\title{
Convenio UNU-Uniandes: Alianza para fortalecer el aprendizaje de temas energéticos en América Latina ${ }^{1}$
}

\author{
UNU-Uniandes Agreement: Alliance to strengthen learning in energy related issues in Latin Americas
}

A finales de la década de los setenta y principios de los ochenta, las facultades de Ingeniería y de Economía de la Universidad de los Andes analizaron el tema del manejo de la energía en todas sus formas, conscientes de la problemática que representaba el uso eficiente y eficaz de los recursos energéticos para el país y la región. Por ello, organizaron seminarios y cursos para sacar adelante proyectos en esta área. Poco a poco, la Universidad fue teniendo reconocimiento en el tema y se logró un convenio con la Universidad de Naciones Unidas (UNU) para impartir un programa internacional de entrenamiento en sistemas y tecnologías energéticas. Cuarenta años después, encontramos nuevamente el tema en el orden del día, razón por la cual, la Revista de ingeniería quiere rescatar la historia de este programa que marcó un hito en América Latina para el aprendizaje de asuntos energéticos.

\section{Antecedentes}

Según consta en las actas de consejo de la Universidad de los Andes, desde 1980 las facultades de Ingeniería y de Economía estaban interesadas en crear un programa de estudios energéticos en la Universidad. Para ese momento, Uniandes contaba con un grupo de investigación en tecnología apropiada y, con laboratorios de modelos y de maquinaria hidráulica, dedicados en parte al estudio de aspectos energéticos. En 1982, Uniandes recibe una invitación de la UNU para hacer parte de un grupo de instituciones de varios países dedicadas a la investigación y a la docencia en el tema de energía. Cabe mencionar que entre 1982 y 1983 se dictaron seminarios sobre fuentes y recursos energéticos y tecnologías de conversión y transporte, aspectos económicos y financiamiento del sector energético, marco institucional y jurídico del manejo de los recursos energéticos en Colombia, entre otros. Además, con el apoyo de Colciencias, se organizó el seminario "nuevas tecnologías energéticas, energías renovables y mercado internacional”.

Estos antecedentes le permitieron a la Universidad presentar, en 1983, una propuesta a la UNU para trabajar en conjunto en temas energéticos. Rafael Beltrán, último coordinador del programa, afirma: "nosotros en esa época teníamos una cierta fortaleza en el tema, había varios profesores de ingeniería eléctrica y mecánica que trabajaban los temas de fuentes renovables. Esa fue la circunstancia por la cual se dio el convenio con la UNU” (comunicación personal, 12 de noviembre de 2015). La idea era darles la oportunidad a personas de otros países de América Latina de venir y aprender del trabajo de los ingenieros y economistas uniandinos.

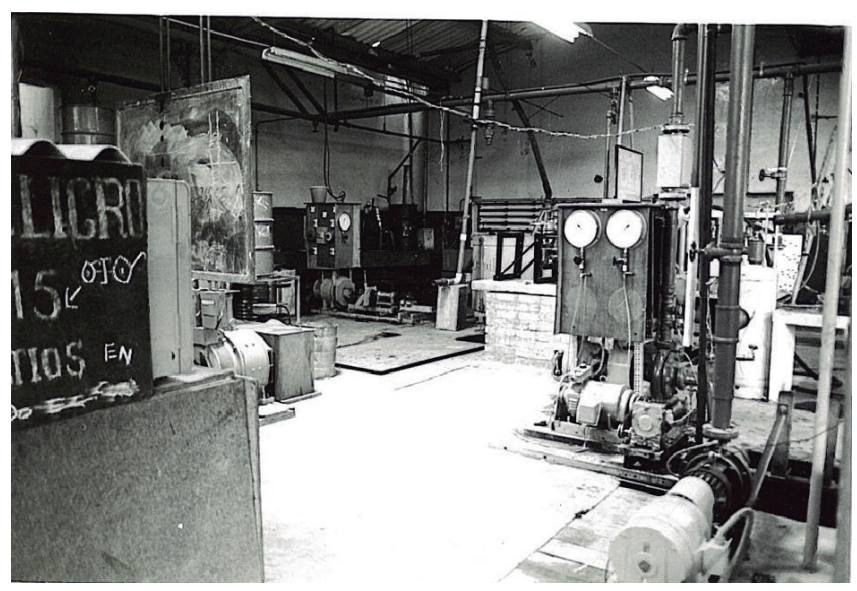

Laboratorio de máquinas hidráulicas.

Fuente: Archivo particular, Universidad de los Andes.

\section{Firma DEL CONVENIO}

Tal como relata Álvaro Pinilla, profesor participante en este programa, "la UNU tiene como política la financiación de programas en determinados temas en distintos lugares del mundo" (comunicación personal, 30 de noviembre de 2015). En ese objetivo, se inscribe la postulación de Uniandes para ser sede del programa de entrenamiento en sistemas y tecnologías energéticas para América Latina. Cabe aclarar que había entidades educativas en otros países interesadas también en la realización de este trabajo.

En aquella época, el vicerrector de la UNU, con sede en Tokio, era Miguel Urrutia, egresado uniandino y muy cercano a Édgar Reveiz, decano de la Facultad de Economía de Uniandes. Gracias a este lazo se empezó a gestar el convenio para que la UNU y Uniandes fueran aliadas en

${ }^{1}$ La memoria fue escrita por María Paula Méndez Penagos a partir de las entrevistas realizadas a Rafael Beltrán, Álvaro Pinilla, Fernando Navas, Luis Fernando Sanz y Javier Gho. 
temas académicos. Sin embargo, también es importante resaltar la participación de otras personas, como Andrés Meleg, vicedecano de la Facultad de Ingeniería, y Álvaro Torres, profesor del departamento de Ingeniería Eléctrica. Ellos también mostraron interés en la creación de este programa. Además, según cuenta Luis Fernando Sanz, primer coordinador del programa, “fue fundamental la ayuda del profesor Fernando Navas en este proceso ya que, él fue el ideólogo del programa” (comunicación personal 18 de diciembre de 2015). Dadas todas estas circunstancias, la Universidad de los Andes presentó su propuesta basada, según Pinilla, “en adquirir una visión tanto técnica como económica de la implementación de nuevas soluciones en energías renovables" (comunicación personal, 30 de noviembre de 2015). De esta manera, logró ser elegida como sede para la realización del programa de entrenamiento e investigaciones en sistemas y tecnologías energéticas con la UNU. El convenio se firmó en 1984 y al año siguiente se inició con la primera promoción.

El convenio consistía en otorgar un número de becas para profesionales latinoamericanos que estuvieran interesados en profundizar sus conocimientos en temas energéticos. La UNU se hacía cargo de todos los gastos de movilidad a Colombia de estas personas y de los costos académicos (matrículas, profesores, materiales, entre otros). Sin embargo, según recuerda Beltrán, “una de las condiciones de la UNU era que no podía haber colombianos en el programa” (comunicación personal, 12 de noviembre de 2015).

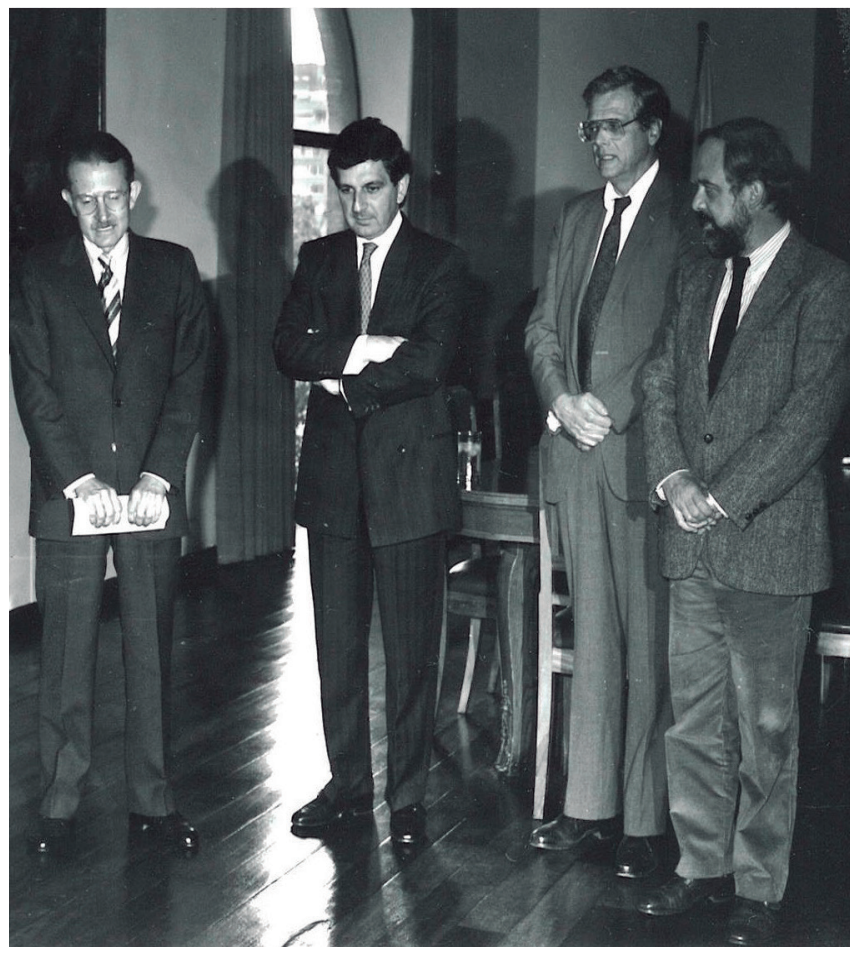

Arturo Infante, Juan M. Caicedo, Miguel Urrutia, Ernesto Guhl Nannetti. Fuente: Archivo particular, Universidad de los Andes.

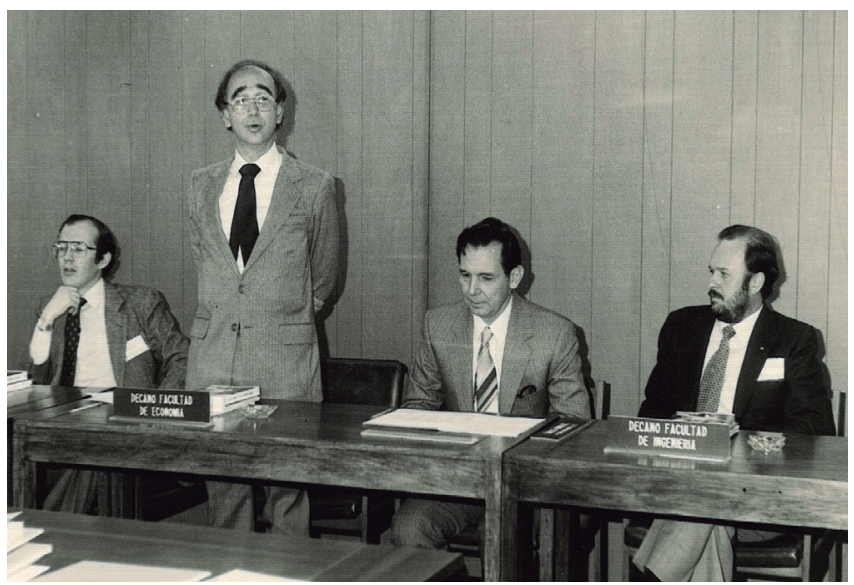

Édgar Reveiz (de pie) y Andrés Meleg (derecha). Fuente: Archivo particular, Universidad de los Andes.

\section{REQUisitos DE INGRESO AL PROGRAMA}

El programa estaba diseñado para profesionales que tuvieran experiencia en temas relacionados con fuentes energéticas renovables. Por esta razón, se enviaba la convocatoria a entidades del sector energético para que personas que estuvieran en el medio pudieran aprovechar esta oportunidad. De igual manera, se contactaba a las universidades latinoamericanas para que los profesores que estuvieran haciendo investigaciones en este campo pudieran participar. El objetivo principal era garantizar que los becarios al regresar a sus países de origen pudieran poner en práctica todo lo aprendido en Colombia.

Los profesores de Uniandes viajaban a los distintos países para entrevistar a los candidatos y poder determinar si eran aptos o no para ingresar al programa. Además, se hablaba con el jefe inmediato, según relata Sanz, "para conocer la utilidad futura del programa UNU-Uniandes en el desarrollo profesional del candidato" (comunicación personal, 18 de diciembre de 2015). Navas recuerda que en ocasiones "no era fácil encontrar gente que estuviera activa en temas de energías renovables, por lo que se buscaban personas que mostraran cierto interés en esos temas y pudieran ser semilla para estudios globales en energía en sus países de origen. En esa época, pocas escuelas de ingeniería en América Latina hacían estudios relacionados con el tema de energía” (comunicación personal, 10 de diciembre de 2015). Este programa podía ser un incentivo para que luego se crearan grupos de investigación que trabajaran en esto.

Se trató de un programa interdisciplinar en el que participaron tanto ingenieros como contadores públicos y economistas. Los participantes venían de todos los países de América del Sur, exceptuando Brasil, y de América Central y México. De hecho, Sanz recuerda que "estudiantes de universidades alejadas de las capitales de Perú y Ecuador valoraban mucho la posibilidad de hacer parte de este 
programa porque llevaban una serie de conocimientos para impulsar líneas de trabajo y de investigación en esos países” (comunicación personal, 18 de diciembre de 2015). Esa interculturalidad también permitió hacer estudios de comparación entre la situación energética que vivía uno y otro país. Entraban por cohorte unas 18 a 20 personas que venían principalmente de empresas de servicios, electrificadoras y universidades. El programa duraba once meses y durante ese tiempo los participantes permanecían en Colombia con el auspicio de la UNU.

\section{ESTRUCTURA DEL PROGRAMA}

El objetivo del programa en entrenamiento e investigaciones en sistemas y tecnologías energéticas, como queda consignado en el Catálogo general, era:

"buscar la actualización y profundización teórica y práctica de los participantes en sistemas y tecnologías energéticas, proporcionándoles de esta manera nuevas y mejores herramientas para el futuro desarrollo de sus funciones, e incentivando la creación y el fortalecimiento de mecanismos de intercambio de conocimientos y experiencias en el dominio de los sistemas energéticos" (Universidad de los Andes, 1985).

Para ello, se propuso una metodología que integrara aspectos teóricos y prácticos. De esta manera, los participantes veían cursos como: energía, recursos naturales y tecnología, fuentes energéticas no convencionales, energías fósiles y tecnologías, economía energética, modelaje y optimización, evaluación socioeconómica de proyectos energéticos y planeación energética global y en la industria. Además, participaban en talleres, laboratorios y visitas a entidades del sector. Cuenta Pinilla que "traíamos gente de Ecopetrol, de Interconexión Eléctrica S.A. (ISA), el Departamento Nacional de Planeación (DNP), entre otros, para dar conferencias. Además, se hacían visitas a El Cerrejón, el Centro Las Gaviotas en Vichada y a algunas empresas que fabricaban equipos en Bogotá” (comunicación personal, 30 de noviembre de 2015). Esto le daba un enfoque muy interesante al trabajo que se realizaba en el programa.

Para complementar el aprendizaje en estas cuestiones, los participantes tenían que desarrollar trabajos de investigación bajo la asesoría de investigadores de la Universidad. Con estos trabajos, afirma Sanz, "se buscaba que ellos ejercitaran esos instrumentos de estudio y de análisis trabajados durante su estadía en Uniandes" (comunicación personal, 18 de diciembre de 2015). A los estudiantes se les pedían dos tareas específicas: la primera, el análisis de la evolución, la historia y la identificación de prioridades de la planeación energética en sus países de origen; y la segunda, presentar a todo el grupo de estudiantes sus hallazgos para examinar las experiencias buenas y malas en América Latina. Sanz asegura también que "estas actividades enriquecían enormemente a los estudiantes y les permitían profundizar sus conocimientos en balances energéticos, políticas energéticas vigentes, estrategias y planes energéticos de cada país latinoamericano" (comunicación personal, 18 de diciembre de 2015).

Como ejemplo de los proyectos llevados a cabo por los participantes están: Aproximaciones metodológicas para la generación de tecnologías apropiadas, elaborado por Javier Gho de Chile y Thelma Rosa Fonseca de Nicaragua bajo la asesoría del profesor Francisco Rodríguez; Metodología para la evaluación de recursos eólicos, a cargo de Raúl Leyte de México y asesorado por el profesor Álvaro Pinilla; Diseño y construcción de una bomba Humphrey, elaborado por Gilberto Zabala de Ecuador y dirigido por el profesor Rafael Beltrán, y Construcción y evaluación de una riobom$b a$, elaborado por Jorge Pizarro de Chile con la asesoría del profesor Jaime Loboguerrero.

Vale la pena destacar que la mayoría de los participantes de este programa continuaron con su trabajo en estos temas en sus países de origen y han hecho aportes significativos desde su área. Por ejemplo, Javier Gho, uno de los beneficiarios de este programa comenta que "este programa fue uno de los primeros a nivel latinoamericano, por lo que fuimos de la primera generación de especialistas en energía y teníamos conocimientos en energías renovables no convencionales (ERNC) que no tenía casi ningún profesional en Chile” (comunicación personal, 21 de diciembre de 2015). Sobre su experiencia, agrega que "me tocó participar como consultor para los dos primeros grandes proyectos en Chile: el diseño para la electrificación con sistemas fotovoltaicos en la región de Coquimbo (6500 sistemas) y el primer parque eólico 1,95 MW en Aysén” (Gho, comunicación personal, 21 de diciembre de 2015). Está claro que el programa fue fundamental para afianzar proyectos en distintas áreas de energía en toda América Latina.

En cuanto a los profesores que participaban en este programa, había tanto de la Facultad de Economía como de la Facultad de Ingeniería. Algunos nombres representativos son Fernando Acosta, Rafael Beltrán, Mario Castillo, David Ledersnaider, Jaime Loboguerrero, Karen Marie Mokate, Fernando Navas, Álvaro Pinilla, Carlos Puech, Jairo Ramírez, Fernando Cepeda, Guillermo Perry, Francisco Rodríguez, Luis Fernando Sanz, José Eddy Torres y Álvaro Torres, entre otros.

\section{Aportes}

Los beneficios que trajo esta alianza UNU-Uniandes fueron tanto a nivel institucional como académico. Por una parte, como asegura Beltrán "lograron consolidarse estudios en temas energéticos en la universidad y se realizaron proyectos con Colciencias" (comunicación personal, 12 de noviembre de 2015). Otro de los grandes logros fue "haber entrenado tanta gente de excelente nivel para que fortalecieran sus actividades en diversas empresas en América Latina”, 
asegura Pinilla. Esto hizo que la Universidad adquiriera gran reputación a nivel internacional y vinieran expertos de distintos lugares a visitar las instalaciones y aprender sobre el tema.

Por otra parte, como relata Navas, gracias a la realización de este programa en Uniandes se logró conformar un grupo de investigación en energía. Este grupo tenía un enfoque novedoso en sistemas porque "incluía una aplicación de los métodos del famoso profesor Stafford Beer para planeación energética colombiana” (comunicación personal, 10 de diciembre de 2015). Es importante resaltar que este enfoque es el único aplicado a los sistemas energéticos de un país. Esto marcó un hito a nivel internacional.

Adicionalmente, Sanz considera que "este convenio ayudó internamente a convencerse de la importancia de ver el sistema energético de manera integral y darse cuenta de que se trata de un trabajo conjunto entre ingenieros, economistas, matemáticos, abogados, entre otros” (comunicación personal, 18 de diciembre de 2015). No sirve el trabajo aislado y desconectado que cada uno haga. Es fundamental intercambiar experiencias y apoyarse entre todos.

\section{EVOLUCIÓN DEL PROGRAMA}

La Universidad de Naciones Unidas apoyó la realización de este programa internacional durante 1985 y 1986. Sin embargo, Rafael Beltrán se dio cuenta de la necesidad que se tenía en el país en el tema y planteó la posibilidad de que el programa siguiera adelante, con fondos uniandinos, y se diera paso a una especialización. En efecto, así se hizo, se rebautizó el programa y en los dos años siguientes se impartió la especialización en gestión de sistemas energéticos. Aquí ya podían participar estudiantes colombianos interesados en el tema. Vale la pena resaltar que en el convenio UNU-Unian- des los participantes recibían un reconocimiento de parte de la UNU pero no había lugar a título de grado. En cambio, con la creación de la especialización y la aprobación del Ministerio de Educación, los estudiantes recibían un grado como especialistas.

Las cifras son significativas, “el programa graduó a 32 estudiantes extranjeros procedentes de varios países de América Latina y 25 estudiantes nacionales en cuatro promociones (1985, 1986, 1987 y 1988) (Beltrán, 1990). Como se puede ver, hubo gran interés en este programa y los resultados obtenidos fueron importantes. No obstante, con el paso del tiempo, fue decayendo el interés en temas energéticos y la especialización dejó de funcionar porque no había estudiantes suficientes. En opinión de Pinilla, “se espera que ahora con la baja en los precios del petróleo y la dependencia del agua en el caso colombiano, se abran fronteras a otro tipo de fuentes energéticas que puedan suministrar energía eléctrica” (comunicación personal, 30 de noviembre de 2015). Esto hará que quizás el interés en energía vuelva a surgir y un programa como el de UNU-Uniandes pueda revivir y relanzarse.

\section{REFERENCIAS}

Archivo general de la Universidad de los Andes. Bogotá Colombia.

Beltrán, R. (1990). Ingeniería mecánica en la Universidad de los Andes. Bogotá, Colombia.

Facultad de Ingeniería, Departamento de Mecánica. (1986). Noticias del departamento. Mecánica al día, 5(14). Bogotá, Colombia.

Universidad de los Andes. Catálogo general 1985-1986. Bogotá, Colombia. 